\title{
Effects of short-term exercise and energy surplus on hormones related to regulation of energy balance
}

\author{
Todd A. Hagobian, Carrie G. Sharoff, Barry Braun* \\ Department of Kinesiology, Energy Metabolism Laboratory, University of Massachusetts, Amherst, MA 01003, USA
}

\begin{abstract}
Energy surplus raises circulating concentrations of leptin and insulin while lowering plasma ghrelin. Exercise has the opposite effects. The purpose of this study was to determine whether exercise counters the hormonal effects of energy surplus independent of changes in energy balance. To do that, we assessed plasma concentrations of leptin, insulin, and ghrelin at baseline, after overfeeding, and after overfeeding plus exercise. Baseline (B) leptin and insulin concentrations and ghrelin area under the curve were measured during an oral glucose challenge in 9 healthy, active subjects ( 6 male, 3 female) after 2 days in energy balance without exercise. Measurements were repeated after subjects were overfed by $+3213 \pm 849 \mathrm{~kJ} / \mathrm{d}$ for 3 more sedentary days (OF). In the third condition, the same net energy surplus $(+3125 \pm 933 \mathrm{~kJ} / \mathrm{d}) \mathrm{was}$ generated for 24 hours by doubling the overfeeding $(+6284 \pm 1669 \mathrm{~kJ} / \mathrm{d})$ and countering it with a bout of exercise (expenditure $=3063 \pm 803$ $\mathrm{kJ}$ ); and measurements were made the next day (OF + EX). Compared with B, leptin went up (5.8 \pm 8.2 to $7.6 \pm 10.6 \mathrm{ng} / \mathrm{mL}) \mathrm{after} \mathrm{OF}$, but was not significantly higher after OF + EX $(7.1 \pm 10.2 \mathrm{ng} / \mathrm{mL})$. Compared with B, insulin was $+36 \%$ and $+43 \%$ higher after OF and OF + EX, respectively. In contrast, ghrelin area under the curve did not change after OF but was significantly lower $(-14 \%)$ than B or OF after OF + EX (indicating greater suppression). These data suggest that the effect of short-term exercise on fasting leptin and insulin depends on energy balance but the ghrelin response may be partially mediated by effects of exercise independent of energy balance.
\end{abstract}

\section{Introduction}

The importance of physical exercise in regulating appetite, energy balance, and, ultimately, body weight is widely recognized. Exercise alters some key hormones (eg, leptin, insulin, and ghrelin concentrations) that modulate energy balance [1-6]. These responses can occur quickly, even after a single exercise bout of sufficient intensity and/or duration [2,5,7-9]. Prolonged exercise ( $\geq 60$ minutes) with a considerable energy expenditure has been shown to lower leptin (measured 48 hours postexercise) and insulin (12-24 hours postexercise) concentrations [8,9], but shorter exercise bouts ( $<60$ minutes) usually do not [10]. One bout of submaximal exercise may [5] or may not influence total ghrelin concentrations measured up to 4 hours later [11-13]. Because few studies are designed to maintain energy balance by increasing dietary energy to account for exercise energy expenditure, the hormonal response to exercise may actually

\footnotetext{
* Corresponding author. Tel.: +1 413577 0146; fax: +1 4135452906.
}

E-mail address: bbraun@kin.umass.edu (B. Braun). be a response to the concurrent energy deficit. In the rare cases when exercise energy expenditure has been replaced by adding calories to the diet, the effects of exercise on leptin, insulin, and/or ghrelin have been inconsistent and likely depend upon timing of measurement(s), intensity and/ or duration of exercise, subject training status, etc [1,14-21]. Broom et al [14] recently reported that, compared with a noexercise control, a single bout of high-intensity exercise reduced the acylated ghrelin area under the curve (AUC) and suppressed appetite for up to 9 hours when energy balance was maintained.

In contrast with exercise, short-term energy surplus (when dietary energy intake exceeds energy expenditure) has the opposite effect on hormones that regulate energy balance. The hormonal response to energy surplus may promote decreased energy intake and increased energy expenditure. In both rodents and humans, energy surplus raises leptin and insulin concentrations and lowers ghrelin concentrations [22-27]. The combined impact of exercise and energy surplus on the hormonal response has rarely been studied $[21,28]$, although it has relevance from both a basic science 
(regulation of energy intake, expenditure, and body weight) and a practical perspective (to minimize weight gain during periods of energy excess, eg, around the holidays). Van Aggel-Leijssen et al [21] found that a single bout of exercise combined with energy surplus increased plasma leptin amplitude and mean insulin concentrations over 24 hours compared with exercise + energy deficit and exercise + energy balance conditions. We previously reported that a single bout of exercise increased insulin action even in the face of continued overfeeding, suggesting that exercise has an effect on metabolic health independent of energy balance [28]. To date, no studies have assessed ghrelin concentrations in response to combining energy surplus and exercise.

Therefore, the purpose of this study was to determine whether the effects of exercise on hormones related to the regulation of energy balance are manifested even in the face of a considerable energy surplus. We expected that, based on the work described above, there would be an independent effect of exercise on the hormonal responses to short-term energy surplus.

\section{Methods}

This was a short-term intervention study in which all of the subjects performed each of the 3 conditions and served as their own controls. Information on subjects and on general procedures used in this study has been previously reported [28], with the most relevant information described below. Percentage changes in fasting insulin concentrations are mentioned briefly in the Results section because they are important to a full understanding of this study, but the exact values are not mentioned in the text or included in the tables or figures because they have been reported previously [28].

\subsection{Subjects}

Nine healthy subjects ( 6 male, 3 female; $28 \pm 8$ years; $74.5 \pm 13.5 \mathrm{~kg} ; 19 \% \pm 10 \%$ body fat; $54.9 \pm 10.4 \mathrm{~mL} /$ [kg.min]) participated in the study. All were nonsmokers, were in excellent overall health, reported being weight stable for the previous 6 months, and participated in regular aerobic exercise (range, 5-8 h/wk). All women had normal menstrual cycles and were studied in the early follicular phase ( 1 to 4 days) of the menstrual cycle based on the date of menstruation. The study was approved by the institutional review board at the University of Massachusetts Amherst. Verbal and written informed consent was obtained from all subjects.

\subsection{Preliminary tests}

Body composition was assessed using dual-energy x-ray absorptiometry (Lunar, Madison, WI). Subjects then performed a continuous progressive exercise test either on a cycle ergometer (SensorMedics 800; SensorMedics, Yorba Linda, CA) or a treadmill (Life Fitness 9100HR; Life Fitness, Schiller Park, IL) to determine peak oxygen consumption using an online metabolic system (Parvomedics TrueMax 2400; Consentius Technologies, Sandy, UT).

\subsection{Estimated energy expenditure and dietary control}

Before the intervention, all subjects completed a 3-day diet recall to provide an initial estimate of energy intake. Resting energy expenditure (REE) was measured in the morning after an overnight fast using indirect calorimetry. After a 20-minute period of relaxation, subjects sat comfortably in a reclining chair for 20 minutes while expired air was collected using the metabolic measurement system (Parvomedics TrueMax 2400). The measured REE was multiplied by an activity factor from 1.5 to 1.8 dependent upon the subject's individual habitual physical activity (based on physical training logs) to provide an estimate of total energy expenditure. Kien and Ugrasbul [29] reported that energy requirements estimated from the REE and the appropriate activity factor were strongly correlated $(r=0.73)$ with energy requirements measured during 28 days of controlled feeding.

Subjects were provided with all meals throughout the study. The standardized diet consisted of common whole and frozen foods. Subjects were asked to consume discrete meals at certain times of the day (breakfast, lunch, dinner, snacks, etc). Subjects were instructed to consume all food and drinks provided and to return the used containers at the end of each day including any uneaten food. Subjects were also instructed to refrain from caffeine and alcohol during the intervention.

\subsection{Experimental protocol}

An overview of the study design is shown in Fig. 1. Baseline testing was conducted after 2 days in which subjects performed no structured exercise (ie, no physical activity beyond daily living) and maintained energy balance as described above (energy intake $=12088 \pm 3117 \mathrm{~kJ} / \mathrm{d}$, energy expenditure $=12171 \pm 2979 \mathrm{~kJ} / \mathrm{d}$ ). On the morning of day 3, after a 10- to 12-hour overnight fast, a catheter was inserted into a forearm vein; and a fasting blood sample was taken followed by an oral glucose challenge. Subjects ingested $75 \mathrm{~g}$ of glucose (Sun Dex; Fisher Healthcare, Houston, TX) within 5 minutes, and blood samples were collected at 30 and 60 minutes. Subjects were then deliberately overfed by $25 \%$

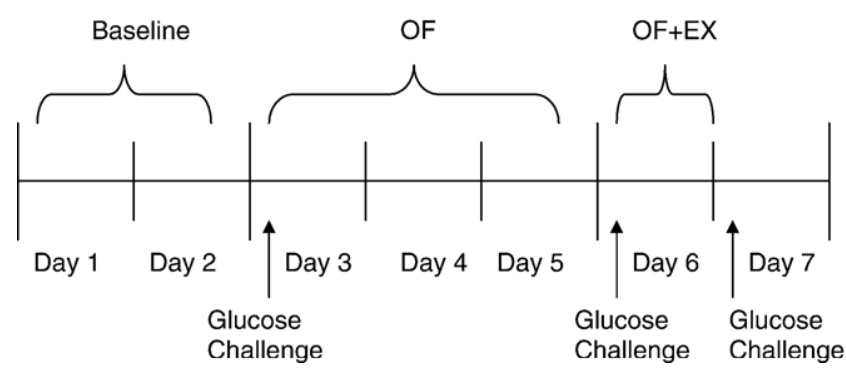

Fig. 1. Overview of the study. 
$(+3213 \pm 849 \mathrm{~kJ} / \mathrm{d})$ above their estimated daily energy needs for the next 3 days while continuing to refrain from structured exercise $(\mathrm{OF}$; energy intake $=15410 \pm 3782 \mathrm{~kJ} / \mathrm{d}$, energy expenditure $=12196 \pm 2996 \mathrm{~kJ} / \mathrm{d}$ ). A second oral glucose challenge was given after the OF period after a 10- to 12-hour overnight fast. After the oral glucose challenge, subjects performed a single bout of exercise comprising $25 \%$ of their estimated daily energy expenditure $(3063 \pm 803 \mathrm{~kJ} / \mathrm{d})$. Subjects completed the exercise protocol on a cycle ergometer or treadmill at $60 \%$ of peak oxygen consumption. To ensure that the exercise intensity and energy expenditure were correct, oxygen consumption was measured during the initial 20 minutes of exercise. Dietary energy was then increased by an additional 50\% above baseline (+6284 \pm $1669 \mathrm{~kJ} / \mathrm{d}$ ) to offset the energy expended during exercise $(\mathrm{OF}+\mathrm{EX} ;$ energy intake $=18376 \pm 4816 \mathrm{~kJ} / \mathrm{d}$, energy expenditure $=15251 \pm 3920 \mathrm{~kJ} / \mathrm{d}$ ). By design, subjects maintained the approximate same energy surplus for OF and $\mathrm{OF}+\mathrm{EX}(+3213 \pm 849 \mathrm{~kJ} / \mathrm{d}$ and $+3125 \pm 933 \mathrm{~kJ} / \mathrm{d}$, respectively). The macronutrient composition of the diet did not change during the intervention because the absolute amounts of ingested carbohydrate, fat, and protein were increased proportionally (56\% carbohydrate, $29 \%$ fat, $15 \%$ protein). The last oral glucose challenge was given the morning after OF + EX after an overnight fast. During the intervention, body mass increased $0.8 \pm 0.6 \mathrm{~kg}$ after OF $(P=$ $.038)$ and a total of $1.1 \pm 0.7 \mathrm{~kg}$ after OF + EX $(P<.001)$ compared with baseline, indicating adherence to the protocol.

\subsection{Biochemical analyses and calculations}

Venous blood samples were collected in sterile syringes and transferred to Vacutainers containing EDTA (Becton Dickinson, Franklin Lakes, NJ) for analysis of leptin and total ghrelin concentrations. Samples were immediately centrifuged at (3000g) for 15 minutes, and plasma was aliquotted into polystyrene tubes and stored at $-80^{\circ} \mathrm{C}$ until analyzed. Plasma insulin concentrations were measured as described previously [28]. Plasma leptin and total ghrelin concentrations were determined by radioimmunoassay (Millipore Research, St Charles, MO). Total ghrelin concentrations were used to calculate AUC during the oral glucose challenge using the trapezoidal method.

Table 1

Fasting plasma leptin and total ghrelin concentrations

\begin{tabular}{llll}
\hline & Baseline & OF & OF + EX \\
\hline Leptin $(\mathrm{ng} / \mathrm{mL})$ & $5.8(8.2)$ & $7.6(10.6)$ & $7.1(10.2)$ \\
$\Delta$ from baseline & & 1.9 & 1.4 \\
$95 \% \mathrm{CI}$ & $(-3.10,-0.03)$ & $(-1.11,1.96)$ \\
Exact $P$ value & & $0.045^{\mathrm{a}}$ & 0.165 \\
Ghrelin $(\mathrm{pg} / \mathrm{mL})$ & $1163(200)$ & $1149(260)$ & $1058(285)$ \\
$\Delta$ from baseline & & -14 & -105 \\
$95 \% \mathrm{CI}$ & $(-129,156)$ & $(-38,247)$ \\
Exact $P$ value & & 0.967 & 0.175 \\
\hline
\end{tabular}

Values are mean $(\mathrm{SD})$ for $\mathrm{n}=9 . \Delta$ indicates change; $\mathrm{CI}$, confidence interval.

${ }^{\text {a }}$ Significantly different than baseline.

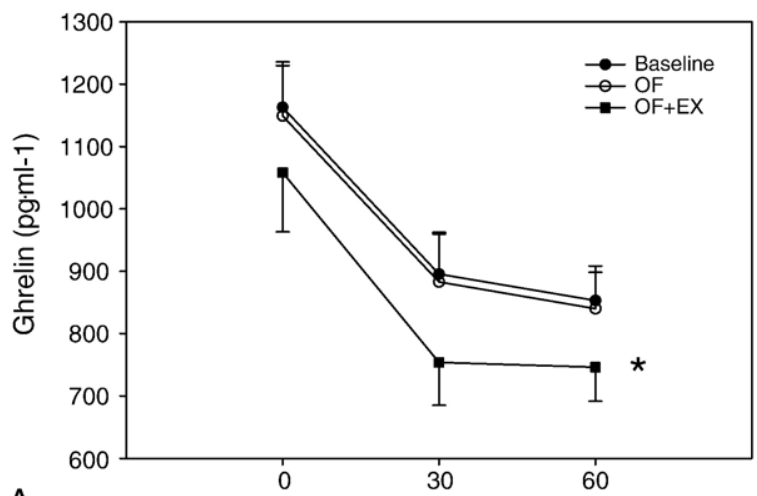

A

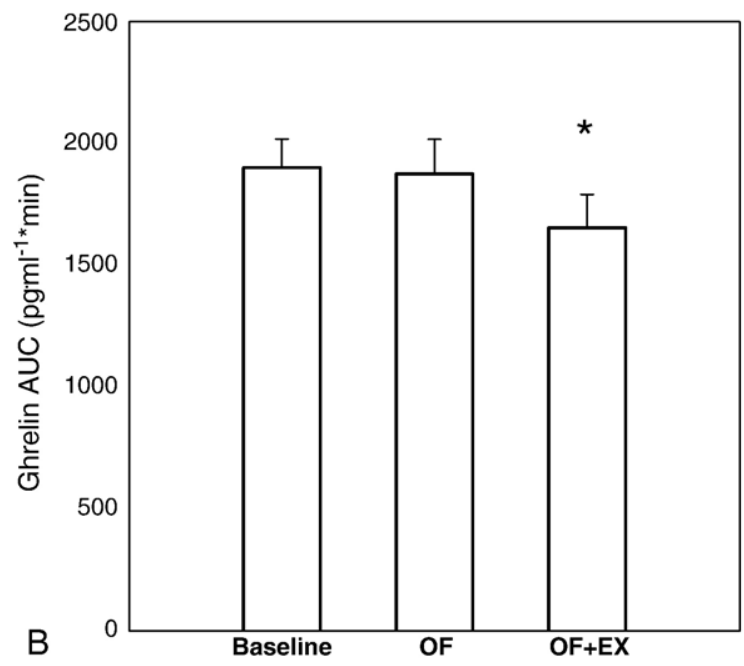

Fig. 2. Total plasma ghrelin response during the 60-minute oral glucose challenge (A) and ghrelin AUC (B) the morning after each period. Values are mean $(\mathrm{SEM})$ for $\mathrm{n}=9$. $* \mathrm{OF}+\mathrm{EX}$ condition significantly different than baseline $(P=.007)$ and $\mathrm{OF}(P=.014)$.

At the time of data collection for this investigation, a commercial kit for active ghrelin was not readily available. Therefore, plasma samples were not treated with an appropriate additive (eg, phenylmethylsulfonyl fluoride $[\mathrm{PMNF}])$ to retard the breakdown of acylated ghrelin, which is very unstable. However, acylated and desacyl ghrelin concentrations are positively correlated [30]. Total ghrelin concentrations have been shown to have short- and long-term responses to changes in energy balance $[18,25,31]$. These results provide confidence that, in a repeated-measures design in which subjects serve as their own controls, total ghrelin concentrations are appropriately responsive to perturbations in short-term energy balance.

\subsection{Statistical analysis}

A commercial software package from SAS (Cary, NC) was used for statistical analysis of data. For all fasting measurements and the ghrelin AUC, a 1-way analysis of variance with a compound symmetric covariate structure was used to test for differences between conditions. For ghrelin concentrations during the oral glucose challenge, a mixedmodel 2-way analysis of variance with repeated measures 
using a compound symmetric covariate structure was used to test for day $\times$ time interactions. By convention, significant differences were defined as $P<.05$. When appropriate, post hoc tests of significance were performed with a Tukey honest significance difference test.

\section{Results}

\subsection{Leptin, insulin, and ghrelin concentrations}

Compared with baseline, fasting leptin concentration was significantly higher after OF $(+31 \%)$ and tended to be higher $(+22 \%)$ after $\mathrm{OF}+\mathrm{EX}$; but this difference between means was not statistically significant $(P>.05$, Table 1$)$. Leptin concentrations were not different between OF and OF + EX. As we previously reported [28], fasting insulin concentration was $36 \%$ higher after OF and $43 \%$ higher after OF + EX compared with baseline. Fasting ghrelin concentrations did not change throughout the intervention (Table 1). The ghrelin response to the glucose challenge (Fig. 2A) and ghrelin AUC (Fig. 2B) were significantly lower $(-14 \%)$ after $\mathrm{OF}+$ EX compared with baseline and OF. To potentially explain the changes in ghrelin response, we looked for correlations between ghrelin AUC and the magnitude of the energy surplus $\left(r^{2}=0.064, P>.05\right)$ or the exercise energy expenditure $\left(r^{2}=0.023, P>.05\right)$; but the coefficients were weak and nonsignificant.

\section{Discussion}

The objective of the current study was to determine whether the effect of exercise on circulating hormones relevant to the regulation of energy balance is independent of changes in energy balance. The main findings were as follows: (1) 3 days of energy surplus increased fasting leptin and insulin concentrations, but had no impact on fasting ghrelin concentrations and (2) with continued energy surplus, a single bout of exercise did not alter fasting concentrations of leptin, insulin, and ghrelin but did lower the ghrelin response to an oral glucose challenge. These data indicate that, whereas fasting concentrations of leptin, insulin, and ghrelin respond to energy balance and are not affected by exercise per se, the ghrelin response to glucose intake is suppressed by exercise independent of changes to energy balance.

Changes in energy balance (deficit, surplus) have a profound impact on hormones that modulate energy intake and expenditure. Diet- or exercise-induced energy deficit decreases leptin and insulin concentrations and increases ghrelin concentrations [17-19] to restore energy balance. In contrast, energy surplus increases fasting leptin and insulin concentrations; but the impact on ghrelin concentrations may depend upon the physiological state (fasting vs mealstimulated) $[25,26,32]$. Ravussin et al [32] found no change in fasting ghrelin concentrations after 100 days of over- feeding in twins. Robertson et al [25] also reported no change in fasting ghrelin but observed a steady decline in ghrelin AUC in response to a high-fat meal during 3 weeks of energy surplus. Although the lack of change in fasting ghrelin that we observed after overfeeding matches prior studies [25,32], we, unlike Robertson et al [25], saw no effect of overfeeding on ghrelin AUC. It is likely that differences in the duration of overfeeding ( 3 days in our study vs 3 weeks for Robertson et al [25]) and/or the macronutrient used to stimulate ghrelin concentrations (glucose or fat challenge) explain the differences. Nevertheless, our data suggest that a compensatory mechanism to lower ghrelin concentrations is not apparent after 3 days of overfeeding.

Previous studies have shown that, when exercise energy expenditure is not replaced, the hormonal response is altered (decreased leptin and insulin, increased ghrelin) in a direction expected to stimulate appetite and restore energy balance [17-19]. When dietary energy is added to replace the exercise energy and restore energy balance, mixed results have occurred [1,14,17-19] depending upon duration/ intensity of exercise, short- vs long-term training, hormone measured, timing of measurement(s), etc. In the one prior study in which dietary energy exceeded exercise energy expenditure to generate an energy surplus, Van AggelLeijssen et al [21] found that 24-hour leptin amplitude and mean insulin concentrations in that condition were higher than when exercise occurred in energy deficit or energy balance conditions. To our knowledge, no previous studies have assessed ghrelin concentrations in response to exercise when combined with energy surplus. In the current investigation, we found that ghrelin AUC was lower after the exercise day compared with baseline and the 3 sedentary overfeeding days, which indicates more suppression of ghrelin from fasting levels by the glucose challenge, suggesting a greater blunting of appetite in the OF + EX condition. A recent well-controlled study showed that a single bout of exercise lowers acylated ghrelin AUC (total ghrelin was not measured) and suppresses appetite, compared with a nonexercise control condition, when energy balance is maintained [14]. Although we did not measure acylated ghrelin, our results are concordant with those data. Thus, our data suggest that, when energy balance is controlled, exercise has an effect on the total ghrelin response to ingested carbohydrate that is independent of changes in energy balance.

Using the same protocol, we previously reported that the insulin AUC was lower $(-20 \%)$ after the overfeeding + exercise day compared with the 3 sedentary overfeeding days [28]. A lower insulin response to the same glucose challenge, which is an estimate of enhanced insulin action, is usually associated with less satiety and increased appetite. In contrast, greater suppression of ghrelin after overfeeding + exercise relative to the other conditions suggests more satiety and decreased appetite. Two possible scenarios may explain the difference in our results. First, insulin and ghrelin may be responding to different mediators. Insulin action is very 
sensitive to exercise, and the insulin response we observed could be a response to a direct or indirect effect of the recent exercise bout. On the other hand, ghrelin may be more responsive to the overall energy intake, which, by design, was considerably higher in the $\mathrm{OF}+\mathrm{EX}$ condition. Because we maintained the same daily energy surplus throughout the intervention (approximately $+3140 \mathrm{~kJ} / \mathrm{d}$ or $+750 \mathrm{kcal} / \mathrm{d}$ ), the cumulative energy surplus after the exercise day $(4$ days $=$ $+12760 \mathrm{~kJ} / \mathrm{d}$ or $+3050 \mathrm{kcal} / \mathrm{d}$ ) was higher than the last overfeeding day ( 3 days $=+8495 \mathrm{~kJ} / \mathrm{d}$ or $+2030 \mathrm{kcal} / \mathrm{d})$. Therefore, by extending the period of overfeeding (and actually increasing total energy intake on the OF + EX day), we may have biased the conditions toward a lower ghrelin response. Both animal and human data support the idea of lower ghrelin concentrations and suppressed appetite with longer periods of overfeeding lasting 1 to 3 weeks $[25,26]$.

Alternatively, the ghrelin response may be a normal consequence of a single prolonged exercise bout rather than the extra day of energy surplus. In previous studies, total or acylated ghrelin concentrations have declined $[5,14]$ after a single bout of exercise; and appetite is suppressed after exercise [4]. Because there was no correlation between ghrelin AUC and either absolute quantity of energy intake or exercise energy expenditure, we cannot rule out either possibility as playing a role in lowering the ghrelin response to glucose with combined exercise and overfeeding.

A limitation of the current investigation is that total ghrelin concentrations were measured rather than acylated ghrelin. Recent evidence indicates that acylation is necessary to bind to the growth hormone secretagogue receptor (now termed ghrelin receptor [33]) within the brain and other tissues and is more biologically active in the regulation of energy homeostasis [34]. Recently, Broom et al [14] showed that acylated ghrelin AUC was lower after a single bout of exercise, whereas most [11-13], but not all [5], other studies have shown no effect of exercise on total ghrelin concentrations. Because acylated ghrelin accounts for such a small portion (10\%-20\%) of total ghrelin [35,36], it is possible that the acylated ghrelin response in these other exercise studies (and in the current study) was obscured and that any changes were underestimated. To more fully understand the impacts of exercise and/or energy balance on the regulation of ghrelin, it will be important to assess both total and acylated ghrelin concentrations.

In summary, we found that exercise and energy surplus had no impact on fasting leptin, insulin, and total ghrelin concentrations relative to overfeeding alone, implying that exercise has no effects independent of energy balance. In contrast, a single bout of exercise combined with energy surplus lowered the total ghrelin response to a glucose challenge, suggesting some metabolic regulation by exercise per se. Further studies in which exercise and energy balance are systematically manipulated are required to tease apart the contributions of exercise and energy balance to the regulation of energy intake and expenditure. From a practical perspective, these results suggest that energy surplus is a more powerful stimulus than a single exercise bout in terms of the impact on hormones critical to the regulation of body weight.

\section{Acknowledgments}

We would like to thank all the volunteers for their time and participation in this investigation. We also thank Brooke Hasson, MS; Steve Malin, MS; Rebecca Hasson, MS; Kaila Holtz; and Kirsten Granados for help with aspects of the study. This investigation was supported by American Diabetes Association Junior Faculty grant 7-04-JF-10.

\section{References}

[1] Black SE, Mitchell E, Freedson PS, Chipkin SR, Braun B. Improved insulin action following short-term exercise training: role of energy and carbohydrate balance. J Appl Physiol 2005;99:2285-93.

[2] Essig DA, Alderson NL, Ferguson MA, Bartoli WP, Durstine JL. Delayed effects of exercise on the plasma leptin concentration. Metabolism 2000;49:395-9.

[3] Torjman MC, Zafeiridis A, Paolone AM, Wilkerson C, Considine RV. Serum leptin during recovery following maximal incremental and prolonged exercise. Int J Sports Med 1999;20:444-50.

[4] King NA, Burley VJ, Blundell JE. Exercise-induced suppression of appetite: effects on food intake and implications for energy balance. Eur J Clin Nutr 1994;48:715-24.

[5] Vestergaard ET, Dall R, Lange KH, Kjaer M, Christiansen JS, Jorgensen JO. The ghrelin response to exercise before and after growth hormone administration. J Clin Endocrinol Metab 2007;92:297-303.

[6] Jurimae J, Hofmann P, Jurimae T, et al. Plasma ghrelin responses to acute sculling exercises in elite male rowers. Eur J Appl Physiol 2007; 99:467-74.

[7] Dostalova I, Bartak V, Papezova H, Nedvidkova J. The effect of shortterm exercise on plasma leptin levels in patients with anorexia nervosa. Metabolism 2007;56:497-503.

[8] Olive JL, Miller GD. Differential effects of maximal- and moderateintensity runs on plasma leptin in healthy trained subjects. Nutrition 2001;17:365-9.

[9] Zhang JQ, Ji LL, Fogt DL, Fretwell VS. Effect of exercise duration on postprandial hypertriglyceridemia in men with metabolic syndrome. J Appl Physiol 2007;19:19.

[10] Kraemer RR, Chu H, Castracane VD. Leptin and exercise. Exp Biol Med (Maywood) 2002;227:701-8.

[11] Kraemer RR, Castracane VD. Exercise and humoral mediators of peripheral energy balance: ghrelin and adiponectin. Exp Biol Med (Maywood) 2007;232:184-94.

[12] Dall R, Kanaley J, Hansen TK, et al. Plasma ghrelin levels during exercise in healthy subjects and in growth hormone-deficient patients. Eur J Endocrinol 2002;147:65-70.

[13] Kallio J, Pesonen U, Karvonen MK, et al. Enhanced exercise-induced GH secretion in subjects with Pro7 substitution in the prepro-NPY. J Clin Endocrinol Metab 2001;86:5348-52.

[14] Broom DR, Stensel DJ, Bishop NC, Burns SF, Miyashita M. Exercise induced suppression of acylated ghrelin in humans. J Appl Physiol $2007 ; 8: 8$.

[15] Dirlewanger M, Di Vetta V, Giusti V, Schneiter P, Jequier E, Tappy L. Effect of moderate physical activity on plasma leptin concentration in humans. Eur J Appl Physiol Occup Physiol 1999;79:331-5.

[16] Fox AK, Kaufman AE, Horowitz JF. Adding fat calories to meals after exercise does not alter glucose tolerance. J Appl Physiol 2004;97:11-6.

[17] Hilton LK, Loucks AB. Low energy availability, not exercise stress, suppresses the diurnal rhythm of leptin in healthy young women. Am J Physiol Endocrinol Metab 2000;278:E43-9. 
[18] Leidy HJ, Gardner JK, Frye BR, et al. Circulating ghrelin is sensitive to changes in body weight during a diet and exercise program in normal-weight young women. J Clin Endocrinol Metab 2004;89: 2659-64.

[19] Leidy HJ, Dougherty KA, Frye BR, Duke KM, Williams NI. Twentyfour-hour ghrelin is elevated after calorie restriction and exercise training in non-obese women. Obesity (Silver Spring) 2007;15: 446-55.

[20] Hickey MS, Houmard JA, Considine RV, et al. Gender-dependent effects of exercise training on serum leptin levels in humans. Am J Physiol 1997;272:E562-6.

[21] van Aggel-Leijssen DP, van Baak MA, Tenenbaum R, Campfield LA, Saris WH. Regulation of average $24 \mathrm{~h}$ human plasma leptin level; the influence of exercise and physiological changes in energy balance. Int J Obes Relat Metab Disord 1999;23:151-8.

[22] Chin-Chance C, Polonsky KS, Schoeller DA. Twenty-four-hour leptin levels respond to cumulative short-term energy imbalance and predict subsequent intake. J Clin Endocrinol Metab 2000;85: 2685-91.

[23] Dirlewanger M, di Vetta V, Guenat E, et al. Effects of short-term carbohydrate or fat overfeeding on energy expenditure and plasma leptin concentrations in healthy female subjects. Int $\mathrm{J}$ Obes Relat Metab Disord 2000;24:1413-8.

[24] Lindqvist A, de la Cour CD, Stegmark A, Hakanson R, Erlanson-Albertsson C. Overeating of palatable food is associated with blunted leptin and ghrelin responses. Regul Pept 2005;130: 123-32.

[25] Robertson MD, Henderson RA, Vist GE, Rumsey RD. Plasma ghrelin response following a period of acute overfeeding in normal weight men. Int J Obes Relat Metab Disord 2004;28:727-33.

[26] Williams DL, Grill HJ, Cummings DE, Kaplan JM. Overfeedinginduced weight gain suppresses plasma ghrelin levels in rats. J Endocrinol Invest 2006;29:863-8
[27] Kolaczynski JW, Ohannesian JP, Considine RV, Marco CC, Caro JF. Response of leptin to short-term and prolonged overfeeding in humans. J Clin Endocrinol Metab 1996;81:4162-5.

[28] Hagobian TA, Braun B. Interactions between energy surplus and shortterm exercise on glucose and insulin responses in healthy people with induced, mild insulin insensitivity. Metabolism 2006;55:402-8.

[29] Kien CL, Ugrasbul F. Prediction of daily energy expenditure during a feeding trial using measurements of resting energy expenditure, fatfree mass, or Harris-Benedict equations. Am J Clin Nutr 2004;80: 876-80.

[30] Akamizu T, Shinomiya T, Irako T, Fukunaga M, Nakai Y, Kangawa K. Separate measurement of plasma levels of acylated and desacyl ghrelin in healthy subjects using a new direct ELISA assay. J Clin Endocrinol Metab 2005;90:6-9.

[31] Foster-Schubert KE, McTiernan A, Frayo RS, et al. Human plasma ghrelin levels increase during a one-year exercise program. J Clin Endocrinol Metab 2005;90:820-5.

[32] Ravussin E, Tschop M, Morales S, Bouchard C, Heiman ML. Plasma ghrelin concentration and energy balance: overfeeding and negative energy balance studies in twins. J Clin Endocrinol Metab 2001;86: 4547-51.

[33] Davenport AP, Bonner TI, Foord SM, et al. International Union of Pharmacology. LVI. Ghrelin receptor nomenclature, distribution, and function. Pharmacol Rev 2005;57:541-6.

[34] Murphy KG, Bloom SR. Gut hormones and the regulation of energy homeostasis. Nature 2006;444:854-9.

[35] Ghigo E, Broglio F, Arvat E, Maccario M, Papotti M, Muccioli G. Ghrelin: more than a natural $\mathrm{GH}$ secretagogue and/or an orexigenic factor. Clin Endocrinol (Oxf) 2005;62:1-17.

[36] Hosoda H, Doi K, Nagaya N, et al. Optimum collection and storage conditions for ghrelin measurements: octanoyl modification of ghrelin is rapidly hydrolyzed to desacyl ghrelin in blood samples. Clin Chem 2004;50:1077-80. 